Method This was a retrospective study including children aged 2 months - 15 years of age. CRSS were performed using Somnotouch RESP (including Flow, RIPsum, Heart rate and Saturations) alongside standalone pulse oximetry using Masimo Rad97, both Somnotouch RESP and Masimo Rad 97 sleep and wake times were identical. Masimo Rad 97 uses an averaging time of 2-4 seconds compared to 4 seconds for Somnotouch RESP oximeter. Statistical analysis was performed with Graphpad Prims 9 using the Wilcoxon signed rank test.

Results A total of 25 studies were included in the analysis (19 males and 6 females). Table 1 shows the comparison between the oxygen saturation data recorded on Somnotouch RESP integrated oximeter and standalone Masimo Rad 97.

Conclusion Our study shows there is a significant difference in oxygen saturation data obtained by these two different pulse oximeters; these differences are likely to be due to differences in technical specifications and merit further investigation. Our study therefore highlights the need for specialist physiologist review of oximetry studies.

\section{THE ASSOCIATION OF DELTA POWER DURING SLEEP WITH CONCURRENT NOCTURNAL AND NEXT-DAY PAIN: RESULTS FROM A COHORT OF FEMALE PARTICIPANTS WITH TEMPOROMANDIBULAR JOINT PAIN}

${ }^{1}$ Matthew Reid* ${ }^{1}$ Dave Abishek, ${ }^{3}$ Chung Mun, ${ }^{4}$ Darlynn Rojo-Wissar, ${ }^{3}$ ane Phillips, ${ }^{1}$ Luis Buenaver, ${ }^{1}$ Claudia Campbell, ${ }^{1}$ Jennifer Jennifer Haythornthwaite, ${ }^{1}$ Patrick Finan, ${ }^{1}$ Michael Smith. 'Johns Hopkins School of Medicine, Baltimore, USA; ${ }^{2}$ University of Arizona, Pheonix, USA; ${ }^{3}$ University of Maryland, Baltimore, USA; ${ }^{4}$ Brown University, Providence, USA

\subsection{6/bmjresp-2021-bssconf.28}

Introduction Existing data demonstrate reduced delta power during sleep in chronic pain and depressed patients. However, there has been little examination of the relationship between delta power and next-day reports of pain. We tested the extent to which nocturnal (during the concurrent sleep period) and daytime pain reports are associated with delta power during sleep, as well as the extent to which this association is moderated by depressive symptoms. We hypothesised that reduced delta power and SWS would be associated with increased pain, pain catastrophising, and pain sensitivity.

Methods 149 female participants with insomnia and temporomandibular joint pain (TMD) were recruited. We examined nocturnal and daytime measures of pain (pain severity, average pain), pain catastrophizing, and objective pain sensitivity (obtained through quantitative sensory testing (QST)), and calculated relative nocturnal delta $(0.5-3.4 \mathrm{~Hz})$ power using polysomnography. We fit linear regression models correcting for depressive symptom severity, age, and total sleep time, and further examined the moderating effect of depression severity on these measures.

Results reduced delta power was associated with increased average nocturnal pain (Unstandardized $\beta=-17.67, p=0.02$ ), morning pain (Unstandardized $\beta=-15.67, p=0.02$ ), and average next-day pain (Unstandardized $\beta=-16.74, p=0.03$ ). Depression severity did not moderate these relationships. Delta power was not significantly associated with objective pain-sensitivity, nocturnal, or daytime pain catastrophising. However the association between nocturnal pain catastrophising and delta power was moderated by depressive symptom severity ( $p$
$=0.04)$. Simple slopes analysis revealed that when participants had low depressive symptoms ( $<13$ CESD), increased delta power significantly predicted reduced nocturnal pain catastrophising.

Discussion These findings demonstrate that delta power during sleep is associated with both nocturnal and daytime experience of pain in patients with TMD. In patients with TMD and low depressive symptoms, reduced delta power was associated with increased nocturnal pain catastrophising.

\section{SLEEP QUALITY, MENTAL HEALTH, AND CIRCADIAN RHYTHMS DURING COVID LOCKDOWN - RESULTS FROM THE SLEEPQUEST STUDY}

\begin{abstract}
${ }^{1}$ Bhavisha Desai* ${ }^{1}$ Neil Carrigan, ${ }^{1}$ Alfie Wearn, ${ }^{1}$ Jonathan Blackman, ${ }^{2}$ Saba Meky, ${ }^{1}$ James Selwood, ${ }^{3}$ Hugh Piggins, ${ }^{4}$ Nicholas Turner, ${ }^{5}$ Rosemary Greenwood, ${ }^{1}$ Elizabeth Coulthard. ${ }^{1}$ ReMemBr Group, Institute of Clinical Neurosciences, Bristol Medical School, Southmead Hospital, University of Bristol, Bristol, UK, BS10 5NB., UK; ${ }^{2}$ Bristol Brain Centre, Southmead Hospital, North Bristol NHS Trust, Bristol, BS10 5NB, UK; ${ }^{3}$ School of Physiology, Pharmacology and Neuroscience, University of Bristol BS8 1TD, UK; ${ }^{4}$ Population Health Sciences Institute, Bristol Medical School, University of Bristol, Bristol, UK, BS8 1UD., UK; ${ }^{5}$ NIHR Research and Design Service South West, University Hospitals Bristol and Weston NHS Foundation Trust, Education and Research Centre, Level 3, Upper Maudlin Street, Bristol,BS2 8AE, UK
\end{abstract}

\subsection{6/bmjresp-2021-bssconf.29}

Introduction Behavioural responses to COVID-19 lockdown will define the long-term impact of psychological stressors on sleep and brain health. Here, we tease apart factors that help protect against sleep disturbance. We capitalise on the unique restrictions during COVID-19 to understand how time of day of daylight exposure and outside exercise interact with chronotype and sleep quality.

Methods Participants completed our online 'SleepQuest' Study between 29th April 2020- 13th May 2020 and were followed up between 5th November 2020 -2nd December 2020. The SleepQuest survey comprised a set of validated questionnaires probing sleep quality, depression, anxiety, and attitudes towards sleep alongside bespoke questions on the effect of COVID-19 lockdown on sleep, time spent outside and exercising and self-help sleep measures.

Results 3474 people from the UK (median age 62, range 1891) completed the baseline data with 2781 participants followed up. Results showed sleep quality was negatively affected by the first UK lockdown restriction [mean PSQI at baseline 8.12 (2.92)] however from baseline to follow up, sleep quality improved (mean PSQI Difference $=2.21 ; 95 \% \mathrm{CI}=[2.12,2.33$.$] )$ Factors that predicted poor prolonged sleep quality were baseline sleep quality $(\mathrm{P}<0.001)$, anxiety $(\mathrm{P}<0.001)$ and attitudes towards sleep $(\mathrm{P}<0.01)$. Better sleep quality was associated with going outside and exercising earlier, rather than later in the day. However, the benefit of being outside early is driven by improved sleep in 'owl' $(\mathrm{p}=0.0002)$ and not 'lark' $(p=0.27)$ chronotype, whereas the benefit of early exercise (inside or outside) did not depend on chronotype.

Discussion We have provided evidence to suggest anxiety and dysfunctional attitudes towards sleep predicted poorer prolonged sleep quality. Defining the interaction between chronotype, mental health and behaviour will be critical for targeted lifestyle adaptations to protect brain health through current and future crises. 